\title{
Determinants of successful transthoracic defibrillation and outcome in ventricular fibrillation
}

\author{
G W N Dalzell, A A J Adgey
}

\begin{abstract}
Objective-To examine factors determining defibrillation success and outcome in patients with ventricular fibrillation.
\end{abstract}

Design-Observational prospective study of age, sex, transthoracic impedance, site of cardiac arrest, ventricular fibrillation duration and amplitude, primary or secondary ventricular fibrillation, aetiology, number of shocks to correct ventricular fibrillation, and drug treatment.

Setting-A teaching hospital and a mobile coronary care unit with a physician.

Patients-70 consecutive patients (50 male, 20 female) mean age $66 \cdot 5$ years.

Interventions-Before the first countershock was administered transthoracic impedance using a $30 \mathrm{kHz}$ low amplitude AC current passed through 8 $\mathrm{cm} / 12 \mathrm{~cm}$ self-adhesive defibrillator electrode pads applied in the anteroapical position was measured. The first two shocks were $200 \mathrm{~J}$ delivered energy (low energy) and further shocks of $360 \mathrm{~J}$ (high energy) were given if required.

Main outcome measures-Countershock success and outcome from ventricular fibrillation.

Results and conclusions-First shock success was significantly greater in inhospital arrests $(37 / 53)$ than in out-ofhospital arrests (5/17) and in those receiving antiarrhythmic treatment $(13 / 15,86.7 \%) v(27 / 51,52.9 \%)$. Transthoracic impedance was similar in those who were successfully defibrillated with one or two $200 \mathrm{~J}$ shocks $(98.7(26) \Omega$ ) and those who required one or more $360 \mathrm{~J}$ shocks (91.4 (23) $\Omega$ ). Success rates with two $200 \mathrm{~J}$ shocks were similar in those patients with "high" transthoracic impedance (that is, $>115 \Omega$ ) and those with transthoracic impedance $(\leqslant 115 \Omega)$ $(8 / 12(67 \%) \quad v \quad 44 / 58 \quad(76 \%))$. Fine ventricular fibrillation was significantly more common in the patients with a transthoracic impedance of $>95 \Omega(41 \%$ (13/32)) than in those with a transthoracic impedance $\leqslant 95 \Omega(13 \%(5 / 38))$. Death during arrest was significantly more common in patients who needed high energy shocks $(14 / 18(78 \%))$ than in those who needed low energy shocks (16/ $52(31 \%))$. Multiple regression analysis identified ventricular fibrillation with an amplitude of $\geqslant 0.5 \mathrm{mV}$, age $\leqslant 70$ years, and arrests that needed $\leqslant$ two shocks for defibrillation, in rank order as independent predictors of survival to discharge.

Transthoracic defibrillation remains a cornerstone of modern cardiological practice. Its more widespread application should improve the mortality from ischaemic heart disease, but further research into defibrillation techniques is essential to improve its efficacy. Many factors are thought to influence the success of ventricular defibrillation, but their relative importance is uncertain. ${ }^{1}$

Defibrillation occurs when an adequate intracardiac current depolarises a critical mass of myocardium. ${ }^{2}$ The electrical circuit formed during defibrillation includes the impedance of the two electrode/electrolyte/tissue interfaces and that of the intervening tissues. The most variable component of the electrode-subject circuit is the impedance of the tissues of the thorax, which to a large extent influences the current that will flow through the myocardium.

The importance of transthoracic impedance as a determinant of intracardiac current flow $^{3}$ and the development of methods to predict the transthoracic impedance in advance of the shock $^{4}$ suggested that the assessment of its clinical role was relevant to present cardiological practice. Some previous studies of transthoracic impedance, however, have not assessed other factors that may influence defibrillation success and some combined results for different arrhythmias. ${ }^{56}$ We have previously reported the influence of transthoracic impedance on the energy required for cardioversion of atrial fibrillation. ${ }^{7}$ In the present prospective study we assessed the relative importance of all factors including transthoracic impedance in the determination of defibrillation and survival in patients with ventricular fibrillation.

\section{Patients and methods}

Data were collected on consecutive patients in whom cardiac arrest due to ventricular fibrillation occurred within or outside hospital. There were 70 consecutive cardiac arrests in which the initial rhythm was ventricular fibrillation in 50 male and 20 female patients (mean age 66.5 years (range 45-86)). In 52 acute myocardial infarction (a typical history of chest pain with an increase in cardiac enzymes 
to twice the normal concentration and serial electrocardiographic changes) was diagnosed. The site of infarction was anterior in 26, inferior in 16, other or undetermined in seven, and left bundle branch block in three. Four patients with documented ischaemic heart disease and no recent infarction developed ventricular fibrillation during the course of another illness: hypokalaemia, severe burns, pulmonary oedema, and after anaesthesia. Four patients had a history of proven ischaemic heart disease or suggestive chest pain before collapse and had sustained an out-of-hospital arrest and died before a definite diagnosis could be made. Of these 60 patients, 24 had had a previous myocardial infarction. Of the remaining 10 , all of whom died during the initial arrest, the aetiology of the arrest was chronic airflow obstruction in one, pulmonary embolism in one, and no information was available in the other eight because they had no prior history of ischaemic heart disease and no necropsies were performed.

Thirty four patients sustained ventricular fibrillation in the hospital coronary care unit, 19 patients had ventricular fibrillation in another part of the hospital, and 17 had ventricular fibrillation out of hospital. Three of those who had ventricular fibrillation out of hospital developed ventricular fibrillation after the arrival of the mobile coronary care unit which was summoned because of chest pain.

Primary ventricular fibrillation was defined as ventricular fibrillation occurring in the absence of cardiac failure or hypotension, while secondary ventricular fibrillation occurred in the presence of one or both of these complications. Patients who collapsed with ventricular fibrillation outside hospital with "instantaneous" death who were previously well and who had no previous history of cardiac disease were classed as primary ventricular fibrillation.

The duration of arrhythmia before countershock was estimated by the physician managing the arrest. Coarse ventricular fibrillation was defined as having an amplitude $\geqslant 0.5 \mathrm{mV}$ and fine ventricular fibrillation had an amplitude $<0.5 \mathrm{mV}$. Previous treatment with antiarrhythmic drugs, digoxin, or $\beta$ adrenergic blockers was recorded.

Self-adhesive electrocardiogram defibrillator pads (R2 Corporation) were used with a $12 \mathrm{~cm}$ diameter pad placed under the right clavicle adjacent to the right upper sternum and an $8 \mathrm{~cm}$ pad covering the cardiac apex. This anteroapical position was used for all patients with cardiac arrest. Standard defibrillators were modified to measure transthoracic impedance before delivery of the countershock while the defibrillator was being charged. A $30 \mathrm{kHz}$ low amplitude $(100 \mu \mathrm{A})$ current was passed through the chest via the electrode pads and the resultant voltage developed was proportional to the impedance. We have previously shown that this technique accurately predicts the actual transthoracic impedance for a defibrillatory countershock. ${ }^{8}$ Three defibrillators were thus modified and their accuracy was checked daily against test resistances. One defibrillator was placed in the coronary care unit, and another on the cardiac arrest trolley, which was used throughout the hospital complex, and the third was used for the management of cardiac arrests out of hospital by a physician manned mobile coronary care ambulance. Transthoracic impedance results obtained before the first countershock were recorded for each cardiac arrest. Only the first episode of ventricular fibrillation during each cardiac arrest was included in the analysis of transthoracic impedance because transthoracic impedance is known to decrease with successive shocks. ${ }^{910}$

The effect of pad pressure on transthoracic impedance ${ }^{11}$ was eliminated by pressing the adhesive pads firmly in position and not touching them during the delivery of countershock. It was possible to check the accuracy of pad position after cardiac arrest in the survivors because the edge of the electrode invariably left an erythematous ring on the patient's skin. One patient in whom the placement of electrode pads was deemed to have been suboptimal was excluded from the analysis.

Patients whose condition was initially stable when they were first seen by the mobile coronary care unit, but in whom ventricular fibrillation subsequently developed before arrival at the hospital, were included in the outof-hospital group for analysis of data on site of arrest.

For attempted defibrillation the first shock was $200 \mathrm{~J}$ (delivered) followed by a second shock of $200 \mathrm{~J}$ if the first was unsuccessful. If two low energy shocks were unsuccessful we used shocks of $360 \mathrm{~J}$. Defibrillation was defined as the electrical conversion of ventricular fibrillation to any other rhythm or to asystole. The success or failure of the first $200 \mathrm{~J}$ shock was noted as was the total number of shocks required to correct the first episode of ventricular fibrillation. The outcome was also noted: died during the arrest, survived the arrest but died later during the same hospital admission, or survived to be discharged.

\section{Results}

A single shock of $200 \mathrm{~J}$ was successful in $60 \%$ (42/70), and two $200 \mathrm{~J}$ shocks had a cumulative success rate of $74 \cdot 3 \%(52 / 70)$. All patients were defibrillated at least once. Of the 70 patients, 30 $(43 \%)$ died during the arrest, $13(18.6 \%)$ survived but died later during their hospital admission, and $27(38.6 \%)$ survived to be discharged from hospital.

\section{AGE AND SEX}

There was no difference in success of two $200 \mathrm{~J}$ shocks in patients aged 70 years or less $(32 / 47$, $68.1 \%)$ and those who were older than $70(20 /$ $2387.0 \%$ ), $p>0.05$. Older patients, however, were less likely to survive the arrest itself and to reach discharge from hospital. A larger proportion of the younger patients $(44.7 \%(21 / 47))$ survived to discharge than of those over 70 years $(26 \%(6 / 23))(p=0.04)$. First shocks were equally successful in men and women (men 30/50, 60\% $v$ women $12 / 20,60 \%$ ). There was no significant difference in transthoracic impedance between men (mean (SD) 91.8 
(21) $\Omega$ ) and women (96.7 (SD) $29 \Omega$ ) $(\mathrm{p}>0.05)$.

\section{TRANSTHORACIC IMPEDANCE}

The mean (SD) transthoracic impedance of this group was 93.3 (22) $\Omega$ (range 38-137). Univariate analysis showed that the first shock or two low energy shocks were as successful in those with an initial transthoracic impedance of $>95 \Omega$ as in those with transthoracic impedance of $\leqslant 95 \Omega$ (fig 1 ). Nor was there any difference between those with a transthoracic impedance $>115 \Omega($ mean $(1 S D)$ ) and $\leqslant 115 \Omega$ (fig 1). The mean transthoracic impedance for those who were successfully defibrillated with one or two $200 \mathrm{~J}$ shocks was 98.7 (26) $\Omega$, compared with $91.4(23)$ for those who required one or more shocks of $360 \mathrm{~J}$ for defibrillation ( $p>0.05$ ). But outcome was not as good in patients with a transthoracic impedance of $>115 \Omega$. Only $8 \cdot 3 \%(1 / 12)$ of them survived to leave hospital, compared with $44.8 \%(26 / 58)$ in the group with a transthoracic impedance $\leqslant 115 \Omega(\mathrm{p}<0.05)$. There was a significant relation between transthoracic impedance and ventricular fibrillation amplitude. Of the patients with a transthoracic impedance $>95$ $\Omega, 41 \%(13 / 32)$ had fine ventricular fibrillation compared with only $13 \%(5 / 38)$ of those with a transthoracic impedance $\leqslant 95 \Omega(p=0.02)$. There was no significant difference in transthoracic impedance between those with out-ofhospital ventricular fibrillation and those with ventricular fibrillation occurring within hospital.

SITE OF CARDIAC ARREST, DURATION OF VENTRICULAR FIBRILLATION, AND AMPLITUDE OF VENTRICULAR FIBRILLATION

Ventricular fibrillation occurring in hospital was associated with a significantly higher first shock success rate than ventricular fibrillation occurring outside hospital $(37 / 53(69 \cdot 8 \%) v$ $5 / 17(29.4 \%) \mathrm{p}=0.007)$. The location at which the arrest occurred also determined survival. The death rate was lower and the discharge rate higher for arrests that occurred in the coronary care unit group than for arrests that happened elsewhere in the hospital, and survival was poorest for arrests out of hospital (fig 2).

Ventricular fibrillation lasted $>3$ minutes in $19 / 70$ patients and $\leqslant 3$ minutes in the remain-
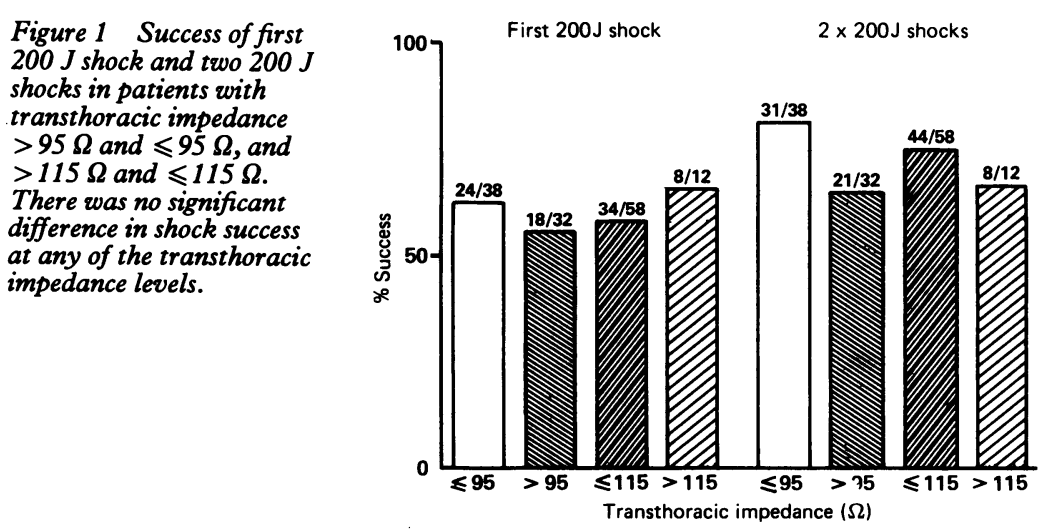

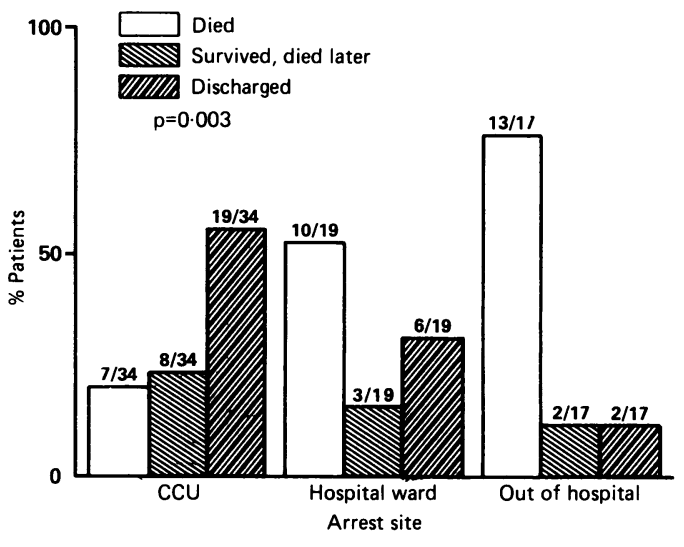

Figure 2 Arrest site and outcome of ventricular fibrillation. Outcome for patients with ventricular fibrillation out of hospital was significantly worse than for those who developed ventricular fibrillation within the coronary care unit (CCU) or another ward within the hospital.

ing 51 . The duration of ventricular fibrillation was not significantly associated with success of a $200 \mathrm{~J}$ shock, but was significantly associated with outcome. Only $2 / 19(10.5 \%)$ patients in whom ventricular fibrillation lasted $>3 \mathrm{~min}$ utes survived to discharge, compared with $25 / 51(49 \%)$ in whom it lasted $\leqslant 3$ minutes $(\mathrm{p}<0.001)$. Ventricular fibrillation lasting more than three minutes was more likely to be fine amplitude $(11 / 19,57.9 \%)$ than ventricular fibrillation lasting $\leqslant$ three minutes $(7 / 51$, $13.7 \%)(\mathrm{p}<0.001)$.

A single shock was successful in $61.5 \%$ $(32 / 52)$ of patients with coarse ventricular fibrillation and $55.6 \%(10 / 18)$ in those with fine ventricular fibrillation $(p>0.05)$. Similarly, two $200 \mathrm{~J}$ shocks had a cumulative success rate of $76.9 \%(40 / 52)$ in patients with coarse ventricular fibrillation compared with $66.7 \%$ $(12 / 18)$ in those with fine ventricular fibrillation $(p>0.05)$. Only two patients with fine ventricular fibrillation survived the arrest, and none survived to be discharged from hospital.

\section{PRIMARY AND SECONDARY VENTRICULAR}

FIBRILLATION

Secondary ventricular fibrillation occurred in 18 patients; it was not more difficult to defibrillate than primary ventricular fibrillation. The first shock success rates $(13 / 18(72.2 \%) v$ $29 / 52(55 \cdot 8 \%)$ and cumulative success of two $200 \mathrm{~J}$ shocks $(14 / 18(77 \cdot 8 \%) v 38 / 52(73 \cdot 1 \%))$ were similar in secondary and primary ventricular fibrillation ( $p>0.05)$.

\section{AETIOLOGY OF VENTRICULAR FIBRILLATION}

Ventricular fibrillation was caused by acute myocardial infarction in $74.3 \%$ of patients $(52 / 70)$. This group had a cumulative defibrillation success rate with two $200 \mathrm{~J}$ shocks of $75 \cdot 0 \%(39 / 52)$ that was similar $(72 \cdot 2 \%(13 / 18))$ to that in other patients $(p>0.05)$. None the less, survival was significantly better for this subgroup, both for survival from the arrest initially $(35 / 52(67 \cdot 3 \%) v 5 / 18(27 \cdot 8 \%))$ and for discharge from hospital $(24 / 52(46 \cdot 2 \%) v$ $3 / 18(16 \cdot 7 \%)), p=0 \cdot 01$. 
ventricular fibrillation was related to outcome. Of those requiring low energy shocks (one or two $200 \mathrm{~J}$ shocks to correct ventricular fibrillation) $46 \cdot 2 \%(24 / 52)$ survived to discharge compared with only $16.7 \%$ (3/18) of those requiring high energy - that is, $>2$ shocks $(p=0.05)$. A significantly higher proportion of patients in the high energy group died during the arrest $(14 / 18(77 \cdot 8 \%) v 16 / 52(30.8 \%)$ $\mathrm{p}=0.002$ ).

Those who received more than five shocks had a less favourable outcome. None of the eight patients given more than five shocks survived the arrest whereas $64.5 \%(40 / 62)$ of those given five or fewer than five shocks survived $(p=0.002)$

\section{DRUG TREATMENT}

There were data on digoxin treatment for 67 patients, and on antiarrhythmic agents and $\beta$ adrenergic blockers for 66 patients. Eighteen patients were not taking any of these drugs, four were taking $\beta$ blockers only, one digoxin only, and one antiarrhythmic agents only. The remaining 42 patients were taking various combinations of drugs. Individual antiarrhythmic agents were not identified because the small numbers of each would have rendered analysis difficult. Most of the 15 patients who were taking antiarrhythmic agents before ventricular fibrillation developed were treated with either lignocaine or mexiletine. One patient had been receiving intravenous bretylium tosylate and four amiodarone. Twenty four patients were taking digoxin and seven $\beta$ adrenergic blocking agents. Previous treatment with digoxin or $\beta$ adrenergic blocking agents had no significant effect on shock success or outcome. Antiarrhythmic treatment was associated with improved first shock success rates (13/15 $(86.7 \%) v 27 / 51(52 \cdot 9 \%) \mathrm{p}=0.04)$.

\section{MULTIPLE REGRESSION ANALYSIS}

A multiple regression analysis was performed to assess the predictive value of the following factors in determining first shock success: age, sex, transthoracic impedance, arrest site, duration of ventricular fibrillation, ventricular fibrillation amplitude, type of ventricular fibrillation (primary or secondary), aetiology of ventricular fibrillation, and drug treatment. Multiple regression identified arrest site in hospital as the most important predictor of first shock success. Treatment with antiarrhythmic agents was identified as the second most important predictive factor. No other variables contributed significantly.

For outcome from ventricular fibrillation, a second multiple regression analysis was performed. The same factors plus the total number of shocks required for defibrillation were entered. The factors of greatest significance in predicting discharge from hospital in rank order of importance were ventricular fibrillation amplitude $\geqslant 0.5 \mathrm{mV}$, age $\leqslant 70$ years, and $\leqslant 2$ shocks to correct ventricular fibrillation.

\section{Discussion}

In this group of patients with ventricular fibrillation, defibrillation success with $200 \mathrm{~J}$ shocks was not related to transthoracic impedance. Multiple regression analysis did not select transthoracic impedance as an important predictor of first shock success. We found that low energy shocks were as successful in patients with a high transthoracic impedance, which was arbitrarily defined as mean $+1 \mathrm{SD}$-that is $>115 \Omega$, as they were in patients with a transthoracic impedance of $\leqslant 115 \Omega$. This accords with a prospective analysis of defibrillation performed in 183 patients where the initial shock was usually $200 \mathrm{~J} .{ }^{12}$ There was no difference in transthoracic impedance between those who defibrillated and those who did not. ${ }^{12}$ This study did not assess energy requirements and included patients who developed ventricular fibrillation late in the resuscitation, generally after an initial brady-asystolic arrest with defibrillation failing in a high percentage of patients. ${ }^{12}$ Geuze and de Feijter also suggested that transthoracic impedance was not a useful predictor of defibrillation success when low energy shocks were used. ${ }^{13}$ Aylward et al investigated electrode-chest wall coupling agents during canine ventricular defibrillation and found that higher transthoracic impedance did not alter the success of countershock. ${ }^{14}$

It has been suggested that transthoracic impedance is an important determinant of defibrillation at energies lower than $200 \mathrm{~J}$. Kerber et al found that patients with a transthoracic impedance of $>97 \Omega$ had a reduced success rate for defibrillation with $\leqslant 100 \mathrm{~J}$ shocks. ${ }^{5}$ However, this effect was not seen with shocks of $\geqslant 200 \mathrm{~J}$. This study was performed in only 25 patients with ventricular fibrillation and no information was given on the duration of ventricular fibrillation or the amplitude of ventricular fibrillation. In a later study the same group suggested that shocks of $100 \mathrm{~J}$ were less effective in patients with a transthoracic impedance of $\geqslant 70 \Omega$ than in those with a transthoracic impedance of $<70 \Omega .^{6}$ In this study also, most patients did not have ventricular fibrillation, and of those who did, most developed the arrhythmia in the electrophysiological laboratory. ${ }^{6}$ Ventricular defibrillation in these situations may not be representative of true emergency defibrillation. Furthermore, in this study those patients who developed ventricular fibrillation elsewherethat is in locations other than the electrophysiology or cardiac catheterisation laboratories and required "emergency" defibrillation were excluded from receiving an initial $100 \mathrm{~J}$ shock and were treated with an initial shock of $200 \mathrm{~J}$ regardless of their transthoracic impedance. ${ }^{6}$ Separate transthoracic impedance ranges for patients with different arrhythmias were not given; this is important because the mean (SD) transthoracic impedance for the group was 75 (21) $\Omega$. Our mean transthoracic impedance for ventricular fibrillation patients was significantly higher than this and was similar to that reported by Lerman et al who studied patients who developed ventricular fibrillation during electrophysiological testing. ${ }^{15}$ Thus the conclusions made by Kerber $e t$ 
al are based on a mixed group of patients, of whom only some required emergency defibrillation. ${ }^{6}$ Extrapolation of these results to the clinical setting has to be viewed with caution. We believe that our patients with ventricular fibrillation are representative of the true emergency clinical situation because in all of them ventricular fibrillation occurred spontaneously and not in the catheterisation laboratory. Unlike other studies ${ }^{56}$ we specifically excluded patients with atrial flutter and ventricular tachycardia, because these arrhythmias require a fraction of the energy required to convert atrial and ventricular fibrillation.

We found that patients with ventricular fibrillation had higher mean transthoracic impedance (93 (22) $\Omega$ ) than a group of patients from a previous study undergoing elective cardioversion of atrial fibrillation (69 (16) $\Omega)(p<0.001) .^{7} \quad$ Because transthoracic impedance is dependent on ionic conduction and therefore related to tissue conductivity, it is not surprising that the dramatic haemodynamic and metabolic effects of cardiac arrest can alter transthoracic impedance. Experimental support for this suggestion is provided by Wojtczak who reported that myocardial tissue resistivity increased with hypoxia. ${ }^{16}$ In addition, Childers et al found that the tissue resistance of ventricular myocardium increased with ischaemia and they suggested this may be the result of cell swelling, intercellular uncoupling at gap junctions, and membrane disruption. ${ }^{17}$ Also, during a cardiac arrest skin blood flow is reduced and there is an increased resistivity of stationary blood. ${ }^{18}$

Because it is the current that actually defibrillates, it would be more satisfactory to use a method for defibrillation that was independent of transthoracic impedance and delivered a constant current to each patient. We have reported our initial experience with a microprocessor controlled current based defibrillator that titrates energy according to each patient's individual transthoracic impedance. ${ }^{19}$ This defibrillator has a success rate comparable to conventional defibrillators but it delivers significantly less energy and current per shock.

Single factor analysis identified only the site of the cardiac arrest and prior treatment with antiarrhythmic agents as being significantly associated with the success of the first $200 \mathrm{~J}$ shock. These findings were confirmed by multiple regression analysis. Ventricular fibrillation outside hospital was associated with ventricular fibrillation of a longer duration. Though we found no association between the duration of ventricular fibrillation and shock success, others have reported an inverse relation between the duration of ventricular fibrillation and the success of defibrillation. ${ }^{20}$ We did not find that transthoracic impedance was higher in patients who had cardiac arrest out of hospital and we found no relation between transthoracic impedance and the duration of ventricular fibrillation.

The effect of antiarrhythmic drugs on success of the first shock is interesting but difficult to explain because this group included patients taking lignocaine, mexiletine, amiodarone, and bretylium tosylate either singly or in various combinations. Because of the small numbers of patients taking each drug, we did not perform individual analyses. It has been suggested that the effects of drugs on transmembrane sodium and potassium currents explains the alterations in the defibrillation threshold. ${ }^{21}$ Lignocaine produced a small fall $(7 \%)$ in canine transthoracic impedance when it was given with pentobarbital anaesthesia. ${ }^{22}$ Bretylium tosylate increased the ventricular fibrillation threshold, ${ }^{23}$ reduced defibrillation threshold, ${ }^{24}$ and can cause chemical defibrillation. ${ }^{25}$

Univariate analysis showed that survival to discharge was poorer among older patients ( $>70$ years), those with transthoracic impedance $>115 \Omega$, when an initial arrest occurred outside hospital, when ventricular fibrillation lasted more than 3 minutes before attempted defibrillation, when there was fine ventricular fibrillation, when the aetiology of ventricular fibrillation was not myocardial infarction, and when more than two shocks were needed to correct ventricular fibrillation. Multiple regression analysis showed that the factors of greatest significance (in rank order) in predicting discharge from hospital were ventricular fibrillation amplitude $\geqslant 0.5 \mathrm{mV}$, age $\leqslant 70$ years, and the requirement of $\leqslant$ two shocks to correct ventricular fibrillation. Dunn et al reported poorer survival to discharge for those receiving five or more shocks ${ }^{26}$ to correct ventricular fibrillation at the time of the initial arrest. Weaver et al showed that patients who survived had required fewer shocks than patients who died later in hospital $(2.6$ shocks $v$ 3.6) ${ }^{27}$ Defibrillation can impair left ventricular function. ${ }^{28}$ There appears to be an association between survival and shock energy, because we found that those who were defibrillated by one or two $(200 \mathrm{~J})$ shocks had a significantly better survival rate than those who needed more than two shocks (and thus high energy shocks). Campbell et al found that of 11 patients in whom shocks of $200 \mathrm{~J}$ were unsuccessful and were given $400 \mathrm{~J}$ (stored energy) shocks, only five survived to leave hospital. ${ }^{29}$ Higher energy shocks produced a higher incidence of atrioventricular block after defibrillation though survival rates were similar. ${ }^{27}$ The actual energy delivered to a patient for a given selected energy setting of the defibrillator depends on their transthoracic impedance. High impedance patients receive more energy and develop a lower peak current than low impedance patients. We found that $6 / 12$ patients with a transthoracic impedance of $>115 \Omega$ died during the arrest and only one survived to be discharged.

The association of fine ventricular fibrillation with an almost universally poor outcome has been widely reported. ${ }^{30}$ Fine ventricular fibrillation represents a degeneration induced by metabolic changes, and as ventricular fibrillation continues its amplitude decreases. We found a significant association between fine ventricular fibrillation and ventricular fibrillation lasting more than three minutes. Also, patients with a transthoracic impedance of 
$>95 \Omega$ were more likely to have fine ventricular fibrillation than those with a transthoracic impedance of $\leqslant 95 \Omega$. Fine ventricular fibrillation is as easy to defibrillate as coarse ventricular fibrillation-we obtained similar success rates both for first shock and for two $200 \mathrm{~J}$ shocks. This is similar to the results of another study. ${ }^{30}$ The equality of ease of defibrillation accords with the finding that coarse ventricular fibrillation does not reflect greater synchronisation of activity than fine ventricular fibrillation. ${ }^{32}$

Myocardial ischaemia reduced the ventricular fibrillation threshold ${ }^{33}$ but did not seem to increase the defibrillation threshold. ${ }^{34}$ Moderate ischaemia makes the threshold for ventricular fibrillation fall below control values and severe ischaemia produces a ventricular fibrillation threshold higher than that obtained with moderate ischaemia; thus severe ischaemia may make the ventricle less vulnerable to fibrillation. ${ }^{35}$ Ventricular fibrillation with causes other than myocardial ischaemia or infarction is a more sinister development and is usually secondary to other disease processes the severity of which determines the outcome.

1 Crampton R. Accepted, controversial, and speculative aspects of ventricular defibrillation. Prog Cardiovasc D is 1980;23:287-306.

2 Zipes DP, Fischer J, King RM, Nicoll Ade B, Jolly WW. Termination of ventricular fibrillation in dogs by Cardiol 1975;36:37-44.

3 Kugelberg J. The interelectrode electrical resistance at defibrillation. Scand $J$ Thorac Cardiovasc Surg 1972;6:274-7.

4 Geddes LA, Tacker WA, Schoenlein W, Minton M, Grubbs $S$, Wilcox $P$. The prediction of the impedance of the thorax to defibrillating current. Med Instrum 1976;10: thorax

5 Kerber RE, Kouba C, Martins J, Kelly K, Low R, Hoyt R, et $a l$. Advance prediction of transthoracic impedance in human defibrillation and cardioversion: importance of impedance in determining the success of low-energy impedance in determining the suc

6 Kerber RE, Martins JB, Kienzle MG, Constantin L Olshansky B, Hopson R, et al. Energy, current, and success in defibrillation and cardioversion: clinical studies using an automated impedance-based method of energ adjustment. Circulation 1988;77:1038-46.

7 Dalzell GWN, Anderson J, Adgey AAJ. Factors determin ing success and energy requirements for cardioversion of atrial fibrillation: revised version. $O J M e d$ 1991;78:85-95.

8 Dalzell GWN, Magee H, Anderson J, Adgey AAJ. Predicted and actual impedance in cardioversion [Abstract]. Cir culation 1986;74 (suppl II):II-388.

9 Dahl CF, Ewy GA, Ewy MD, Thomas ED. Transthoracic impedance to direct current discharge: effect of repeated countershocks. Med Instrum 1976;10:151-4

10 Geddes LA, Tacker WA, Cabler P, Chapman R, Rivera R, Kidder $H$. The decrease in transthoracic impedance during successive ventricular defibrillation trials. Med Instrum 1975;9:179-80.

11 Kerber RE, Grayzel J, Hoyt R, Marcus M, Kennedy J. Transthoracic resistance in human defibrillation. Circula tion 1981;63:676-82.
12 Kerber RE, Jensen SR, Gascho JA, Grayzel J, Hoyt R, Kennedy J. Determinants of defibrillation: prospective analysis of 183 patients. Am J Cardiol 1983;52:739-45.

13 Geuze RH, de Feijter PJ. Evaluation of transthoracic countershock with initial energy levels up to $200 \mathrm{~J}$ in a countershock with initial energy levels up to 200

14 Aylward PE, Kieso R, Hite P, Charbonnier F, Kerber RE. Defibrillator electrode-chest wall coupling agents: Defibrillator electrode-chest wall coupling agents: Am Coll Cardiol 1985;6:682-6.

15 Lerman BB, DiMarco JP, Haines DE. Current-based versus energy-based ventricular defibrillation: a prospective study. J Am Coll Cardiol 1988;12:1259-64.

16 Wojtczak J. Contractures and increase in internal longitudinal resistance of cow ventricular muscle induced by hypoxia. Circ Res 1979;44:88-95.

17 Childers RW, Cope T, Lyon R, Holland R. Tissue resistance in ventricular ischemia [Abstract]. Circulation 1979;59 and 60 (suppl II):II-110

18 Geddes LA, Baker LE. The specific resistance of biological material-a compendium of data for the biomedical engineer and physiologist. Med Biol Eng 1967;5:271-93.

19 Dalzell GWN, Cunningham SR, Anderson J, Adgey AAJ. Initial experience with a microprocessor controlled current based defibrillator. Br Heart J 1989;61:502-5.

20 Yakaitis RW, Ewy GA, Otto CW, Taren DL, Moon TE. Influence of time and therapy on ventricular defibrillation influence of time and therapy on ventricul

21 Babbs CF. Alteration of defibrillation threshold by antiarrhythmic drugs: a theoretical framework. Crit Care Med 1981;9:362-3.

22 Kerber RE, Pandian NG, Jensen SR, Constantin L, Kieso $\mathrm{RA}$, Melton J, et al. Effect of lidocaine and bretylium on energy requirements for transthoracic defibrillation: experimental studies. J Am Coll Cardiol 1986;7:397-405.

23 Chow MSS, Kluger J, DiPersio DM, Lawrence R, Fieldman A. Antifibrillatory effects of lidocaine and bretylium immediately postcardiopulmonary resuscitation. Am Heart $J$ 1985;110:938-43.

24 Tacker WA, Niebauer MJ, Babbs CF, Combs WJ, Hahn BM, Barker MA, et al. The effect of newer antiarrhythmic drugs on defibrillation threshold. Crit Care Med 1980; 8:177-80.

25 Sanna G, Arcidiacono R. Chemical ventricular defibrillation of the human heart with bretylium tosylate. Am J Cardiol of the human hear

26 Dunn HM, McComb JM, MacKenzie G, Adgey AAJ. Survival to leave hospital from ventricular fibrillation. Am Heart $J$ 1986;112:745-51.

27 Weaver WD, Cobb LA, Copass MK, Hallstrom AP. Ventricular defibrillation-a comparative trial using 175-J and 320-J shocks. N Engl J Med 1982;307:1101-6.

28 Stoddard MF, Labovitz AJ, Stevens LL, Buckingham TA, Redd RR, Kennedy HL. Effects of electrophysiologic studies resulting in electrical countershock or burst pacing on left ventricular systolic and diastolic function. $A m$ Heart $J$ 1988;116:364-70.

29 Campbell NPS, Webb SW, Adgey AAJ, Pantridge JF. Transthoracic ventricular defibrillation in adults. $B M J$ 1977;ii:1379-81.

30 Weaver WD, Cobb LA, Dennis D, Ray R, Hallstrom AP, Copass MK. Amplitude of ventricular fibrillation waveform and outcome after cardiac arrest. Ann Intern waveform and outcom

31 Stults KR, Brown DD, Kerber RE. Ventricular fibrillation amplitude predicts ability to defibrillate [Abstract]. $J \mathrm{Am}$ Coll Cardiol 1987;9:152A

32 Jones DL, Klein GJ. Ventricular fibrillation: the importance of being coarse? J Electrocardiol 1984;17:393-400.

33 Burgess MJ, Abildskov JA, Millar K, Geddes JS, Green LS. Time course of vulnerability to fibrillation after experimental coronary occlusion. Am J Cardiol 1971;27: 617-21.

34 Kerber RE, Pandian NG, Hoyt R, Jensen SR, Koyanagi S, Grayzel J, et al. Effect of ischemia, hypertrophy, hypoxia,
acidosis, and alkalosis on canine defibrillation. Am J Physiol 1983;244:H825-31.

35 Fisher J, Sonnenblick EH, Kirk ES. Increased ventricular fibrillation threshold with severe myocardial ischemia. $\mathrm{Am}$ Heart J 1982;103:966-72. 